\title{
Odontoid metastasis: a potential lethal complication
}

\author{
Faizal Rayan - Cibu Mukundan - D. D. Shukla • \\ R. L. Barrington
}

Received: 28 July 2009/ Accepted: 23 October 2009/Published online: 18 November 2009

(C) Springer-Verlag 2009

\begin{abstract}
Nearly one third of cervical spine metastasis has a primary breast malignancy. Patients with cervical metastasis have higher mortality due to advanced stage of the malignancy. Treatment is palliative to relieve pain, prevent pathological fracture, improve mobility and function, and prolong survival. We describe a 40 -year-old woman with a history of breast cancer who presented with neck and shoulder pain of 1 week duration with no neurological deficit. Following clinical examination, radiographs taken of the cervical spine was normal. Radiographs repeated 3 weeks later revealed a large lytic lesion of the odontoid occupying $70-80 \%$ of the peg. Further investigation including magnetic resonance imaging and bone scan showed no further spinal lesions. She underwent cyclical radiotherapy with complete resolution of the odontoid peg lesion and clinically was asymptomatic at 2 years. Metastatic lesions of the odontoid are atypical, and this case reinforces the necessity of early detection to evade disastrous consequences.
\end{abstract}

Keywords Odontoid $\cdot$ Metastases $\cdot$ Spine $\cdot$ Secondaries . Complication

\section{Introduction}

Spine is the most common site of bone metastasis. The occurrence of cervical spine metastasis is less common than thoracic or lumbar spine metastasis. This spinal

F. Rayan $(\bowtie) \cdot$ C. Mukundan · D. D. Shukla · R. L. Barrington Department of Trauma and Orthopaedics,

Kettering General Hospital, Rothwell road,

15 Moorhouseway, Kettering NN15 7LX, UK

e-mail: rayanmarakkar@yahoo.co.uk metastasis can be osteosclerotic or osteolytic. To recognise spinal metastasis radiographically, $30-50 \%$ of the vertebral body must be involved [1]. Thirty percent of cervical spine metastasis had a primary breast malignancy [2]. Patients with cervical metastasis have a higher mortality due to advanced stage of the malignancy. Although the incidence of skeletal metastasis in breast carcinoma is $30 \%$ with the possibility of prolonged survival, little importance has been given to metastasis of the odontoid process [2]. Odontoid fractures in patients with metastatic carcinoma of the breast are rare. The clinical findings lag behind the radiographic abnormalities. Prompt treatment can relieve symptoms and avoid late sequelae; therefore the recognition of this potentially lethal complication is of prime importance. Treatment is palliative to relieve pain, prevent pathological fracture, improve mobility and function, and prolong survival.

\section{Case report}

A 40-year-old woman presented to the accident and emergency department with neck and shoulder pain of 1 week duration with no radiation and neurological deficit. Following clinical examination, radiographs of the cervical spine were normal. She was discharged home on analgesics. She presented 3 weeks later with increasing intensity of pain. On further review of history, she had been diagnosed with carcinoma of the left breast 7 years earlier, for which she had wide local excision followed by chemotherapy and radiotherapy. She had axillary recurrence after 3 years, for which she had complete remission on chemotherapy and hormonal therapy. Following further recurrence in the left axilla, the patient underwent left mastectomy and axillary clearance. Histology revealed 
high-grade invasive mixed lobular carcinoma and malignant cell infiltration of axillary soft tissue.

Radiographs were repeated, which revealed a large lytic lesion of the odontoid occupying $70-80 \%$ of the peg. As per Harrington's criteria, it was a class II lesion [1]. The cervical spine was immobilised and a computed tomography (CT) scan was performed, which revealed no pathological fracture of the odontoid peg but confirmed the lytic lesion. On further investigations of the spine, including magnetic resonance imaging (MRI) and bone scintigram, no other spinal lesions were noted. She underwent cyclical radiotherapy following review by the oncologist. At a year's follow-up radiographs revealed complete resolution of the odontoid peg lesion and the woman was clinically asymptomatic. Consent was provided by the patient to publish this clinical report.

\section{Discussion}

Vertebral metastases often are asymptomatic and may be discovered only by routine bone scans. Symptoms often develop due to: (1) increase in the size of the lesion within
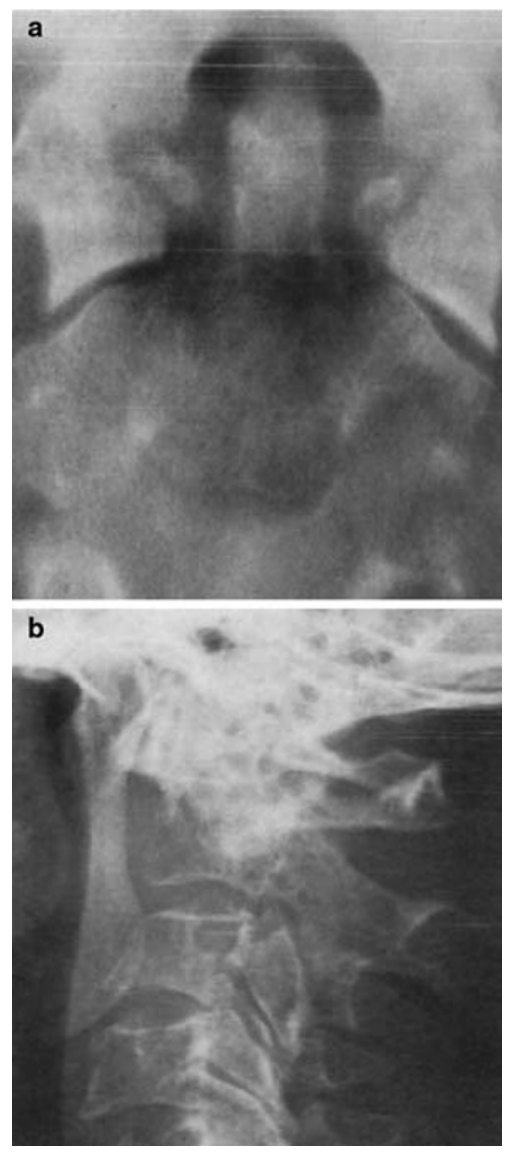

Fig. 1 a, b Anteroposterior (AP) and lateral radiographs of the cervical spine showing a large lytic lesion of the odontoid the vertebral body, which may breach the cortex and invade paravertebral soft tissues, (2) compression of adjacent nerve roots, (3) development of a pathological fracture, (4) development of spinal instability from such a fracture and compression of the spinal cord [1]. Such compression has been reported to occur in approximately $5 \%$ of patients with widespread cancer [3, 4]. Harrington classified patients with spinal metastases into five categories, depending on the extent of neurological compromise or bone destruction, and he recommended treatments according category: class I-no significant neurological involvement; class II-involvement of bone without collapse or instability; class III-major neurological impairment (sensory or motor) without significant involvement of bone; class IV-vertebral collapse with pain due to mechanical causes or instability but without significant neurological compromise; class V-vertebral collapse or instability combined with major neurological impairment [1].

The aim of treatment is to relive pain, prevent development of pathological fracture, improve mobility and
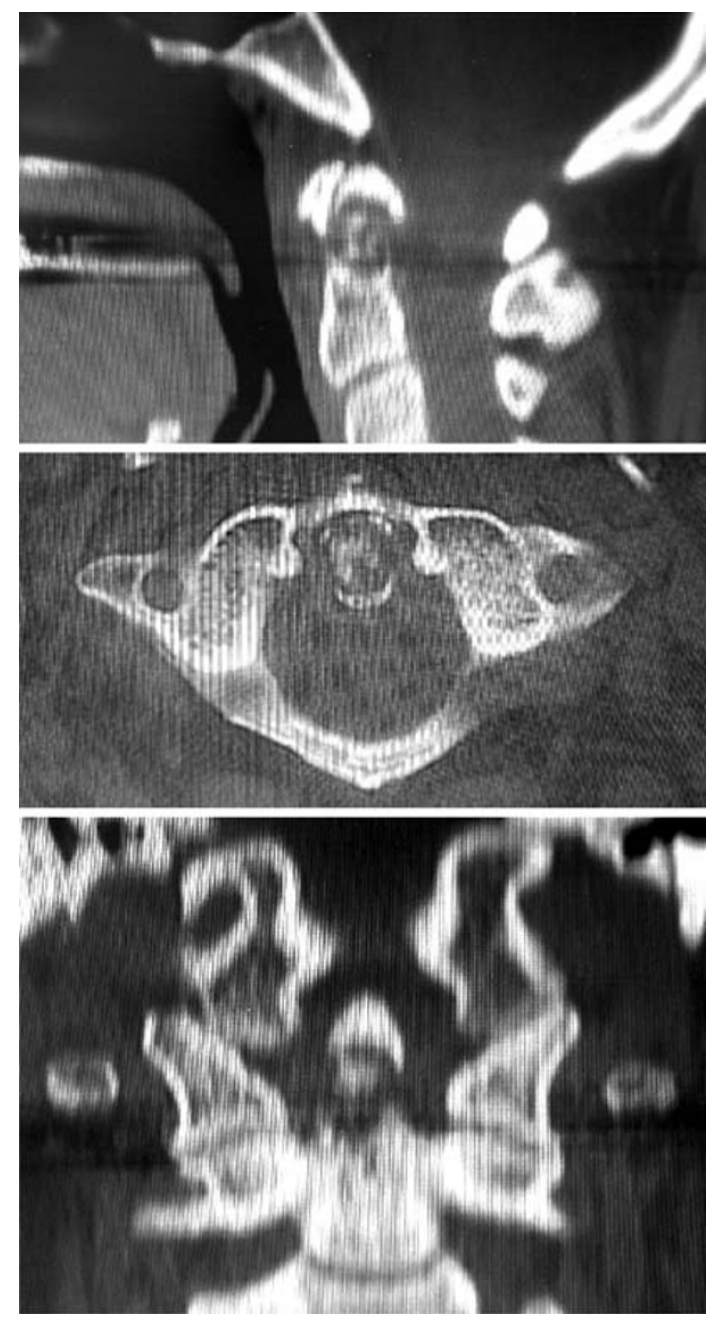

Fig. 2 Computed tomography (CT) showing the odontoid lesion 


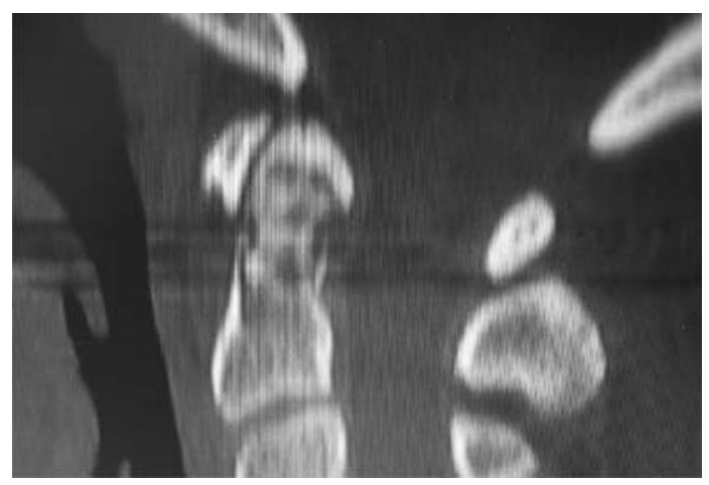

Fig. 3 Computed tomography (CT) showing resolving lesion postradiotherapy

function, and prolong survival. Patients in class I or II generally obtain relief from pain by chemotherapy or hormonal manipulation, and in the absence of success with these modalities, from local irradiation. Radiotherapy is the mainstay of treatment for class III. Surgery is indicated for classes IV and V to prevent or improve neurological dysfunction, to correct a kyphotic deformity, and to relieve intractable pain to improve the quality of life in terminally ill patients. To rationalise surgical treatment, life expectancy is an important aspect. More than three fourths patients who died of breast cancer had bony metastasis $[5,6]$. Two to $7 \%$ of them had cervical spine involvement $[5,6]$. Clinical presentation often lags behind radiological findings, which makes metastasis to the odontoid of great significance despite its rarity. Mortality rate is $>50 \%$ in acute odontoid fractures [7]. Any patient with an acute odontoid fracture is at high risk of neurological complications, as progressive odontoid displacement or minimal fracture may result in spinal cord compression. This could present as early as 6 weeks as late as 60 years after odontoid fracture [8-10].

Metastatic lesions of the odontoid are rare, and in this case report, the lesion was identified before a pathological fracture occurred [2]. Hence, one must be aware of patients presenting with neck pain following a diagnosis of breast cancer, as they are at high risk of developing cervical metastasis, and if they are subjected to any intervention that may require a general anaesthetic, preoperative evaluation is vital, as only a minimal force is required to cause fracture. To prevent this from occurring, the main strategies should be: (1) Continuous surveillance of patients with breast carcinoma for clinical symptoms such as neck pain and stiffness; (2) If patients present with neck pain, adequate views of cervical spine (anteroposterior/lateral and open-mouth view) is mandatory, and if in doubt, CT or MRI is warranted. (3) If patients are subjected to general anaesthetic evaluation of cervical vertebra, using appropriate imaging modalities (X-ray, CT \& MRI) is necessary (Figs. 1, 2, 3).

An appropriate clinical history, detailed examination, and investigation with early intervention can avoid disastrous sequelae in such cases.

Conflict of interest statement The authors declare that they have no conflict of interest related to the publication of this manuscript.

\section{References}

1. Harrington KD (1986) Metastatic disease of the spine. J Bone Joint Surg Am 68:1110-1115

2. Hussein AA, El-Karef E, Hafez M (2001) Reconstructive surgery in spinal tumours. Eur J Surg Oncol 27:196-199. doi: 10.1053/ejso.2000.1079 S0748-7983(00)91079-5 [pii]

3. Boland PJ, Lane JM, Sundaresan N (1982) Metastatic disease of the spine. Clin Orthop Relat Res 169:95-102

4. Constans JP, de Divitiis E, Donzelli R, Spaziante R, Meder JF, Haye C (1983) Spinal metastases with neurological manifestations. Review of 600 cases. J Neurosurg 59:111-118

5. Abrams HL, Spiro R, Goldstein N (1950) Metastases in carcinoma; analysis of 1000 autopsied cases. Cancer 3:74-85

6. Staley CJ (1956) Skeletal metastases in cancer of the breast. Surg Gynecol Obstet 102:683-688

7. Lally JF, Cossrow JI, Dalinka MK (1977) Odontoid fractures in metastatic breast carcinoma. AJR Am J Roentgenol 128:817-820

8. Anderson LD, D'Alonzo RT (1974) Fractures of the odontoid process of the axis. J Bone Joint Surg Am 56:1663-1674

9. Dunbar HS, Ray BS (1961) Chronic atlanto-axial dislocations with late neurologic manifestations. Surg Gynecol Obstet 113:757-762

10. Paradis GR, Janes JM (1973) Posttraumatic atlantoaxial instability: the fate of the odontoid process fracture in 46 cases. J Trauma 13:359-367 